УДК $532.711+66.081 .6$

\title{
Theoretical study of electrolyte diffusion through polarizable nanopores
}

\author{
Ilya I. Ryzhkov* \\ Institute of Computational Modelling SB RAS, \\ Akademgorodok 50-44, Krasnoyarsk, 660036 Russia, \\ Siberian Federal University, \\ Svobodny 79, Krasnoyarsk, 660041, Russia \\ Anton S. Vyatkin ${ }^{\dagger}$ \\ Institute of Computational Modelling SB RAS, \\ Akademgorodok 50-44, Krasnoyarsk, 660036 Russia, \\ Siberian Federal University, \\ Svobodny 79, Krasnoyarsk, 660041, Russia \\ Andrey V. Minakov $\ddagger$ \\ Siberian Federal University, \\ Svobodny 79, Krasnoyarsk, 660041, Russia
}

Received 07.11.2017, received in revised form 28.03.2018, accepted 28.03.2018

The diffusion of binary aqueous electrolytes through nanopores with dielectric as well as conductive surface is investigated theoretically on the basis of Space-Charge model. The latter is extended to the case of polarizable nanopore wall. It is shown that the diffusion of ions with different mobilities generates the electric field, which induces non-uniform surface charge in a polarizable nanopore. It results in charge separation inside the pore and leads to a dramatic enhancement of membrane potential in comparison with a non-polarizable nanopore. The calculations are performed for three aqueous electrolytes based on $\mathrm{KCl}, \mathrm{NaCl}$, and $\mathrm{LiOH}$. The influence of electrolyte type and concentration difference applied across the pore on the ion transport and membrane potential is discussed and analyzed.

Keywords: nanoporous membrane, electrolyte transport, diffusion, induced charge, electric double layer, membrane potential, numerical modelling

\section{Introduction}

When a charged membrane separates two salt solutions of different concentrations, a potential difference between them appears. It results from Donnan equilibrium between diffusion and electric forces at membrane/solution interfaces (Donnan potentials), and electric field generated by diffusion of ions with different mobilities (diffusion potential) [1]. The measurement of membrane potential at zero current is used for characterizing the ionic permselectivity of ion exchange membranes and determining their charge density $[2,3]$.

\footnotetext{
*rii@icm.krasn.ru

†jatkinanton@mail.ru

¥tov-andrey@yandex.ru

(c) Siberian Federal University. All rights reserved
} 
A widely used theoretical approach for describing the membrane potential is the TeorellMeyer-Sievers (TMS) model $[4,5]$. It is based on the assumption that the Debye screening length is larger than the pore size, so the potential and ion concentrations in the pore crosssection are uniform. In addition, the osmotic water transport is neglected. For densely charged membranes, the predictions of TMS model are accurate when the concentration difference across the membrane is small [6].

The Space-Charge (SC) model, which takes into account the radial variation of potential and ion concentrations as well as water transport in cylindrical pores, was first suggested in [7] and revisited recently in [8]. The experimental verification of $\mathrm{SC}$ model was performed by comparing the predicted streaming potential, pore conductivity, and membrane potential at zero current with the measured data [9]. The comparison between SC and TMS model showed that the latter overestimates the value of membrane potential for large surface charge and/or pore size exceeding the Debye length [10].

The TMS and SC models assume that the membrane charge is constant, which is typical for many commercially available membranes. In recent decades, a new class of membranes containing gold nanotubules that span a complete thickness of a porous polymeric support was suggested in $[11,12]$. Using membrane potential measurement at zero current, it was shown that their selectivity can be reversible switched from anion to cation by changing the potential applied to the conductive membrane surface. Theoretical studies of electrolyte transport in nanofiltration membranes with conductive surface were performed in $[13,14]$. To correctly describe the ion transfer in conductive nanopores, the fixed surface potential should be assumed [15]. Note that the electric field generated by moving ions near a conductive surface can induce polarization charges [16,17], which may in turn alter the pore transport characteristics.

Recently, it was shown theoretically and experimentally that the induced charge is responsible for significant enhancement of diffusion potential in membranes with polarizable conductive surface [18-20]. In binary monovalent electrolytes, the diffusion of ions with different mobilities through such membranes generates an electric field, which speeds up the slower diffusing ion and retards the faster diffusing ion. The electric field induces a surface charge on a conductive pore wall, which results in charge separation inside the nanopore. The corresponding Donnan potentials appear at the pore entrance and exit leading to a dramatic enhancement of membrane potential in comparison with uncharged dielectric membrane.

In this work, we theoretically investigate the diffusion of electrolytes through a nanopore with conductive polarizable walls. The influence of electrolyte type and applied concentration difference on the ion transport are discussed and analyzed.

\section{Mathematical model of electrolyte diffusion through a nanopore}

Consider a porous membrane, which separates two reservoirs denoted by $L$ (left) and $R$ (right). The reservoirs contain aqueous solutions of the same monovalent and symmetric (1:1) electrolyte with concentrations $C_{L}$ and $C_{R}$, respectively $\left(C_{L}>C_{R}\right)$. The electrolyte diffuses from the reservoir with a higher concentration to that with a lower concentration. The pressures in the reservoirs are equal, and there is no electric current between them. The potential difference between reservoirs, which develops due to diffusion, is denoted by $\Delta \Phi$.

A membrane is modelled as an array of cylindrical pores of length $L_{p}$ and radius $R_{p}$. The cylindrical coordinates $R$ in radial and $Z$ in axial directions are introduced in a single pore 


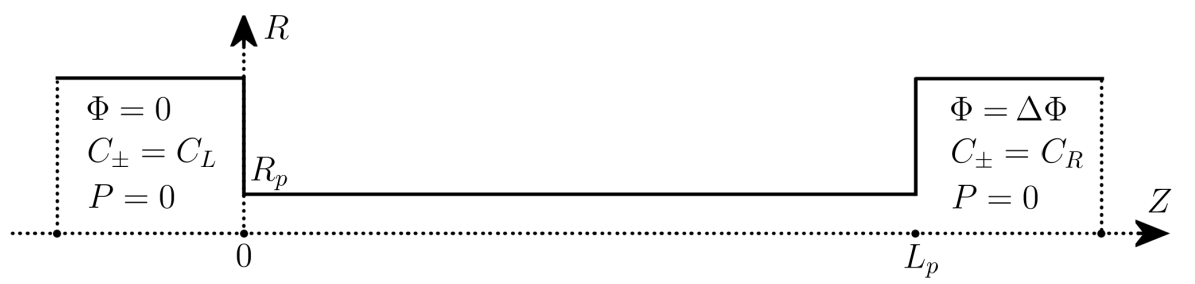

Figure 1: The geometry of a single cylindrical pore.

(Fig. 1). The transport of electrolyte through the pore is characterized by the solution velocity $\boldsymbol{U}=(U, V)$, pressure $P$, cation $C_{+}$and anion $C_{-}$concentrations $\left(\mathrm{mol} / \mathrm{m}^{3}\right)$, and electric potential $\Phi$. These quantities satisfy the system of two-dimensional Navier-Stokes, Nernst-Planck, and Poisson equations $[13,14,17]$. In this work, we consider three types of pores with different boundary conditions on the walls: (1) constant surface charge density $\sigma ;(2)$ constant surface potential $\Phi_{s}$; (3) constant total surface charge $Q$. The total surface charge density is defined by $\bar{\sigma}=Q / 2 \pi R_{p} L_{p}$.

The ions in the pores are transported by convection, diffusion, and migration in electric field. The fluxes of ions are written as

$$
\boldsymbol{J}_{ \pm}=C_{ \pm} \boldsymbol{U}-D_{ \pm} \nabla C_{ \pm} \mp \frac{D_{ \pm} F}{R_{g} T} C_{ \pm} \nabla \Phi
$$

where $D_{ \pm}$are the ion diffusion coefficients, $R_{g}$ is the ideal gas constant, $T$ is the temperature, and $F$ is the Faraday constant. The ion mobilities are calculated as $u_{ \pm}=D_{ \pm} F / R_{g} T$.

Let us introduce dimensionless variables by

$$
\begin{gathered}
R=R_{p} r, \quad Z=L_{p} z, \quad \boldsymbol{U}=\frac{D_{-}}{L_{p}} \boldsymbol{u}, \quad P=C_{0} R_{g} T p, \\
C_{ \pm}=C_{0} c_{ \pm}, \quad \Phi=\frac{R_{g} T}{F} \varphi, \quad \boldsymbol{J}_{ \pm}=\frac{D_{-} C_{0}}{L_{p}} \boldsymbol{j}_{ \pm}, \quad \sigma_{s}=\frac{\sigma}{\varepsilon \varepsilon_{0} R_{g} T / F R_{p}} .
\end{gathered}
$$

Here $\boldsymbol{u}=(u, v)$ and $C_{0}$ is the reference concentration taken as $C_{0}=1 \mathrm{~mol} / \mathrm{m}^{3}$.

In what follows, we will need the quantities averaged over the pore cross-section. The dimensional average axial velocity is defined by

$$
\bar{V}=\frac{2}{R_{p}^{2}} \int_{0}^{R_{p}} V R d R
$$

The average pressure $\bar{P}$, ion concentrations $\bar{C}_{ \pm}$, potential $\bar{\Phi}$, axial ion fluxes $\bar{J}_{ \pm}$, total axial ion flux $\bar{J}=\bar{J}_{+}+\bar{J}_{-}$, and axial ion current $\bar{I}=\bar{J}_{+}-\bar{J}_{-}$are introduced in the same way. The corresponding dimensionless quantities are $\bar{v}, \bar{p}, \bar{c}_{ \pm}, \bar{\varphi}, \bar{j}_{ \pm}, \bar{j}=\bar{j}_{+}+\bar{j}_{-}$, and $\bar{i}=\bar{j}_{+}-\bar{j}_{-}$.

The Space-Charge model is derived from the Navier-Stokes, Nernst-Planck, and Poisson equations by introducing several assumptions appropriate for large aspect ratio pores [8]. The dimensionless potential $\varphi$, ion concentrations $c_{ \pm}$, and pressure $p$ are represented as

$$
\begin{gathered}
\varphi(r, z)=\phi_{v}(z)+\psi(r, z), \quad c_{ \pm}(r, z)=c_{v}(z) \exp (\mp \psi(r, z)), \\
p(r, z)=p_{v}(z)+2 c_{v}(z) \cosh (\psi(r, z)) .
\end{gathered}
$$


Here the ion concentrations satisfy the Boltzmann distribution. The function $\psi$ satisfies the Poisson equation with boundary condition of axial symmetry

$$
\begin{gathered}
\frac{1}{r} \frac{\partial}{\partial r}\left(r \frac{\partial \psi(r, z)}{\partial r}\right)=\frac{c_{v}(z)}{\lambda^{2}} \sinh \psi(r, z), \\
\frac{\partial \psi}{\partial r}(0, z)=0 .
\end{gathered}
$$

The constant surface charge density is imposed by

$$
\frac{\partial \psi}{\partial r}(1, z)=\sigma_{s}
$$

while for the constant surface potential one should write

$$
\psi(1, z)=\varphi_{s}-\phi_{v}(z)
$$

For a polarizable conductive pore wall, the surface potential $\varphi_{s}$ should be determined in order to satisfy the floating boundary condition

$$
\int_{0}^{1} \frac{\partial \psi}{\partial r}(1, z) d z=\bar{\sigma}_{s}
$$

In conditions (5)-(7), $\varphi_{s}, \sigma_{s}$, and $\bar{\sigma}_{s}$ are the dimensionless surface potential, surface charge density, and total surface charge density, respectively.

The relation of the average volume flux $\bar{v}$ (or average axial velocity), average ion flux $\bar{j}=$ $\bar{j}_{+}+\bar{j}_{-}$, and average ion current $\bar{i}=\bar{j}_{+}-\bar{j}_{-}$to the gradients of virtual pressure $p_{v}$, virtual chemical potential $\mu_{v}=\ln c_{v}$, and virtual electric potential $\phi_{v}$ can be written in the form of phenomenological flux-force formalism:

$$
\left(\frac{d p_{v}}{d z}, \frac{d \mu_{v}}{d z}, \frac{d \phi_{v}}{d z}\right)^{T}=L(\bar{v}, \bar{j}, \bar{i})^{T} .
$$

Here $L=-\mathcal{L}^{-1}$ is the symmetric $3 \times 3$ matrix. The coefficients of matrix $\mathcal{L}=\left\{\mathcal{L}_{i j}(z)\right\}$ depend on the function $\psi(r, z)$ and virtual concentration $c_{v}(z)$ according to $[18,19]$

$$
\begin{gathered}
\mathcal{L}_{11}=\frac{1}{8 \alpha}, \quad \mathcal{L}_{22}=2 c_{v} \int_{0}^{1} r(D \exp (\psi(r))+\exp (-\psi(r))) d r- \\
-\frac{16 c_{v}^{2}}{\alpha} \int_{0}^{1}\left[r \cosh (\psi(r)) \ln r\left(\frac{r^{2}}{2} \cosh (\psi(r))-\frac{\lambda^{2}}{4 c_{v}}\left(r \frac{\partial \psi(r)}{\partial r}\right)^{2}\right)\right] d r \\
\mathcal{L}_{33}=-\frac{8 c_{v}}{\alpha} \int_{0}^{1} r\left[\sinh (\psi(r)) \lambda^{2}\left(\psi(r)-\psi_{s}\right)-\frac{\alpha}{4}(D \exp (\psi(r))+\exp (-\psi(r)))\right] d r \\
\mathcal{L}_{12}=\mathcal{L}_{21}=\frac{c_{v}}{\alpha} \int_{0}^{1}\left(r-r^{3}\right) \cosh (\psi(r)) d r, \quad \mathcal{L}_{13}=\mathcal{L}_{31}=\frac{4}{\alpha} \int_{0}^{1} r \lambda^{2}\left(\psi(r)-\psi_{s}\right) d r \\
\mathcal{L}_{23}=\mathcal{L}_{32}=\frac{8 c_{v}}{\alpha} \int_{0}^{1} r\left[\cosh (\psi(r)) \lambda^{2}\left(\psi(r)-\psi_{s}\right)-\frac{\alpha}{4}(D \exp (\psi(r))-\exp (-\psi(r)))\right] d r
\end{gathered}
$$

where $\alpha=\mu D_{-}\left(C_{0} R_{g} T R_{p}^{2}\right)^{-1}, \psi_{s}=\psi(1, z)$, and $\mu$ is the solution dynamic viscosity. The dependence of $\psi$ and $c_{v}$ on $z$ is not explicitly stated in the above formulas. 
The membrane potential is measured at zero current $(\bar{i}=0)$. In this case, system $(8)$ becomes

$$
\frac{d p_{v}}{d z}=L_{11} \bar{v}+L_{12} \bar{j}, \quad \frac{1}{c_{v}} \frac{d c_{v}}{d z}=L_{12} \bar{v}+L_{22} \bar{j}, \quad \frac{d \phi_{v}}{d z}=L_{13} \bar{v}+L_{23} \bar{j} .
$$

The corresponding boundary conditions are derived by assuming equal (zero) pressures and constant concentrations in the reservoirs, and setting the potential at the left reservoir to zero. By putting $\psi(r, z)=0$ in (2), one arrives at

$$
\begin{aligned}
& z=0: \quad p_{v}=-2 c_{L}, \quad c_{v}=c_{L}, \quad \phi_{v}=0, \\
& z=1: \quad p_{v}=-2 c_{R}, \quad c_{v}=c_{R}, \quad \phi_{v}=\Delta \varphi .
\end{aligned}
$$

Here $\Delta \varphi$ is the dimensionless potential difference between the reservoirs (membrane potential).

Let us express $d z=d c_{v} /\left(c_{v}\left(L_{12} \bar{v}+L_{22} \bar{j}\right)\right)^{-1}$ from the second equation in (10) and substitute it into the first and third equations. Integration of resulting equations over the pore length with the help of boundary conditions (11) gives

$$
\begin{gathered}
\int_{c_{L}}^{c_{R}} \frac{L_{11} \bar{f}+L_{12}}{c_{v}\left(L_{12} \bar{f}+L_{22}\right)} d c_{v}+2\left(c_{R}-c_{L}\right)=0, \\
\bar{j}=\int_{c_{L}}^{c_{R}} \frac{d c_{v}}{c_{v}\left(L_{12} \bar{f}+L_{22}\right)}, \\
\phi_{v}\left(c_{v}\right)=\int_{c_{L}}^{c_{v}} \frac{L_{13} \bar{f}+L_{23}}{c_{v}\left(L_{12} \bar{f}+L_{22}\right)} d c_{v},
\end{gathered}
$$

where $\bar{f}=\bar{v} / \bar{j}$ is the fluxes ratio. It follows from (3), (6), (9), and (14) that one can write $\psi=\psi\left(r, c_{v}\right), \phi_{v}=\phi\left(c_{v}\right), L_{i j}=L_{i j}\left(c_{v}\right)$.

The calculation is performed as follows. For a non-polarizable dielectric pore with constant surface charge density $\sigma_{s}$, problem (3)-(5) is solved numerically for a set of successive values $c_{v}=c_{v k}, k=0, \ldots, n$, where $c_{v 0}=c_{L}, c_{v n}=c_{R}$. Then the ratio of fluxes $\bar{f}=\bar{v} / \bar{j}$ is found numerically from (12), and the ion flux $\bar{j}$ is obtained from (13). The potential difference between reservoirs $\Delta \varphi=\phi_{v}\left(c_{R}\right)$ is determined from (14), while the virtual variables are found by integration of (10), (11).

For a polarizable conductive pore with constant total surface charge density $\bar{\sigma}_{s}$, an initial guess for the surface potential $\varphi_{s}$ is set. Here problem $(3),(4),(6)$ is solved for each $c_{v k}$ at fixed $\varphi_{s}$ and $\bar{j}$. Note that $\phi_{v}\left(c_{v 0}\right)=\phi_{v}\left(c_{L}\right)=0$. The value $\phi_{v}\left(c_{v k}\right)$ is found iteratively starting from $\phi_{v}\left(c_{v, k-1}\right)$ and repeating the solution of (3), (4), (6) followed by application of (14). The calculation is performed iteratively to find the fluxes ratio $\bar{f}$ from (12). Then $\bar{j}$ is calculated from (13) and virtual variables are obtained by integration of (10), (11). It allows to find $\psi(r, z)=\psi\left(r, c_{v}(z)\right)$ and finally calculate the distribution of surface charge $\sigma(z)=\partial \psi / \partial r(1, z)$ and the integral in the left-hand side of $(7)$. The whole procedure is iterated to find the surface potential $\varphi_{s}$, with which Eq. (7) is satisfied. This iteration is not required when the surface potential is fixed externally.

The integration of Poisson equation (3) is performed by reducing it to two first-order ODE and applying the Runge-Kutta-Merson method of 5th order starting from $r=\epsilon$ to $r=1$, where $\epsilon$ is close to zero. Here an additional boundary condition $\psi(\epsilon, x)=\psi_{0}$ is required. The value $\psi_{0}$ is determined by the shooting method in order to satisfy boundary condition (5) or (6) at fixed $z$. The initial approximation for it can be found from analytical solution derived in [21]. 
Table 1: The diffusion coefficients of ions.

\begin{tabular}{cccc}
\hline \hline Ions & $\mathrm{K}^{+}, \mathrm{Cl}^{-}$ & $\mathrm{Na}^{+}, \mathrm{Cl}^{-}$ & $\mathrm{Li}^{+}, \mathrm{OH}^{-}$ \\
\hline$D_{+}, 10^{-9} \mathrm{~m}^{2} / \mathrm{s}$ & 1.957 & 1.330 & 1.030 \\
$D_{-}, 10^{-9} \mathrm{~m}^{2} / \mathrm{s}$ & 2.032 & 2.032 & 5.028 \\
$D_{+} / D_{-}$ & 0.963 & 0.654 & 0.195 \\
\hline \hline
\end{tabular}

\section{Results and discussion}

In this work, we consider the diffusion of three aqueous electrolytes on the basis of $\mathrm{KCl}$ and $\mathrm{NaCl}$ salts and $\mathrm{LiOH}$ base through a nanopore. The ion diffusion coefficients and their ratios are given in Table 1. The ratio of diffusion coefficients decreases in the sequence $\mathrm{KCl} \rightarrow \mathrm{NaCl} \rightarrow$ $\mathrm{LiOH}$. To study purely diffusive transport of ions, it is assumed that the membrane is uncharged. For a dielectric nanopore, it means that the surface charge density $\sigma=0$, while for a conductive nanopore, the total surface charge density $\bar{\sigma}=0$. In the latter case, the local surface charge density can be non-zero.

The dimensions of nanopore are taken as $R_{p}=5 \mathrm{~nm}, L_{p}=100 \mu \mathrm{m}$. The parameters used in the calculations are as follows: $T=298.15 \mathrm{~K}, R=8.314 \mathrm{~J} /(\mathrm{mol} \mathrm{K}), F=96485 \mathrm{C} / \mathrm{mol}$, $\varepsilon=78.49, \varepsilon_{0}=8.854 \cdot 10^{-12} \mathrm{~F} / \mathrm{m}$. The dynamic viscosity of ionic solutions is taken as that of water: $\mu=0.888 \cdot 10^{-3} \mathrm{~Pa} \cdot \mathrm{s}$.

The extensive comparison between non-polarizable and polarizable nanopores for different electrolytes is shown in Fig. 2. Let us start with the aqueous $\mathrm{NaCl}$ electrolyte, see Figs. 2 $(\mathrm{e}-\mathrm{h})$. The concentrations at the reservoirs are fixed at $C_{L}=10 \mathrm{mM}$ and $C_{R}=1 \mathrm{mM}$. For a nonpolarizable nanopore, the concentrations of cations and anions coincide. Due to the difference between ion diffusion coefficients $\left(D_{+} / D_{-}=0.654\right)$, the electric field $E=-\nabla \Phi$ develops. It speeds up the slower diffusing cation and retards the faster diffusing anion to make the total ion fluxes equal $\left(\bar{J}_{+}=\bar{J}_{-}\right)$and satisfy the condition of zero current $\left(\bar{I}=\bar{J}_{+}-\bar{J}_{-}=0\right)$. In a polarizable pore, this electric field induces the surface charge, which changes almost linearly from the pore entrance $\left(Z / L_{p}=0\right)$ to the pore exit $\left(Z / L_{p}=1\right)$, while keeping the total surface charge $\bar{\sigma}$ zero, see Fig. 2 (h). It results in the higher concentration of cations (anions) at negatively (positively) charged part of the pore, Fig. 2 (f). The separation of charge induces the Donnan potentials at the pore entrance and exit, which both contribute to the enhancement of membrane potential $\Delta \Phi=\bar{\Phi}\left(L_{p}\right)$ in comparison with non-polarizable pore, Fig. 2 (e). Note that the magnitude of averaged potential inside the pore is smaller for a polarizable case since the induced charge suppresses the electric field in the near-wall region. The separation of ionic charge in the pore also results in osmotic pressure jumps at the pore entrance and exit, see Fig. $2(\mathrm{~g})$. These jumps balance the electric force, which develops in the interfacial regions, where non-zero net charge is subjected to a large electric field. The pressure gradient inside the pore generates osmotic flow in the direction from lower to higher concentration side.

For aqueous $\mathrm{KCl}$ electrolyte, the ion diffusion coefficients almost coincide $\left(D_{+} / D_{-}=0.963\right)$. In this case, the electric field generated by diffusion is much smaller in comparison with $\mathrm{NaCl}$ electrolyte. The induced charge is smaller as well (Fig. 2 (d)), so there is almost no charge separation inside the nanopore (Fig. 2 (b)), and the osmotic pressure jumps essentially decrease (Fig. $2(\mathrm{c})$ ). Although the magnitude of membrane potential is greatly reduced in this case, 

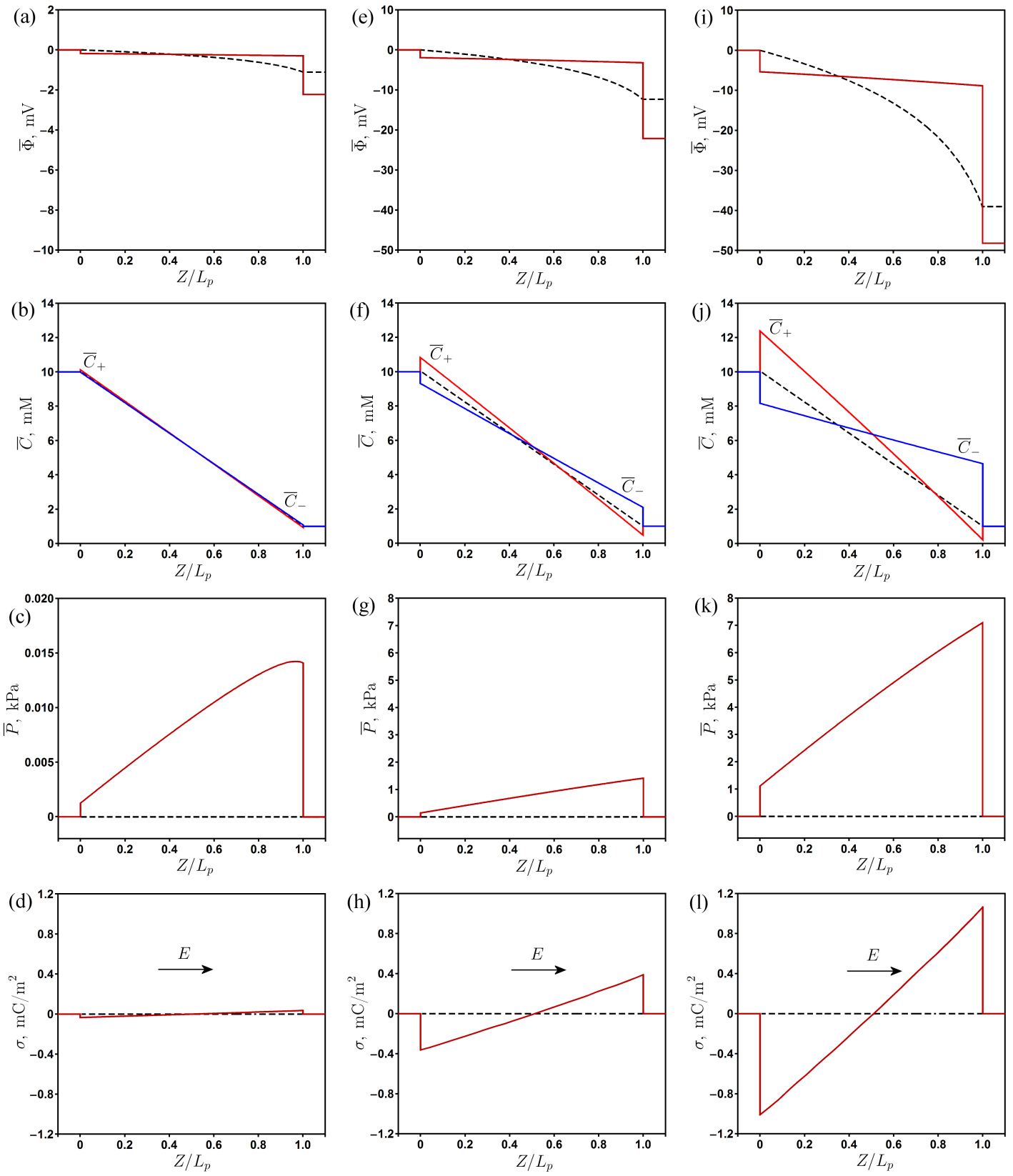

Figure 2: The cross-sectionally averaged potential (a,e,i), concentrations (b,f,j), pressure $(\mathrm{c}, \mathrm{g}, \mathrm{k})$, and surface charged density $(\mathrm{d}, \mathrm{h}, \mathrm{l})$ for aqueous $\mathrm{KCl}(\mathrm{a}-\mathrm{d}), \mathrm{NaCl}(\mathrm{e}-\mathrm{h})$, and $\mathrm{LiOH}$ (i-l) electrolytes. Non-polarizable nanopore (dashed curves) and polarizable nanopore (solid curves). 

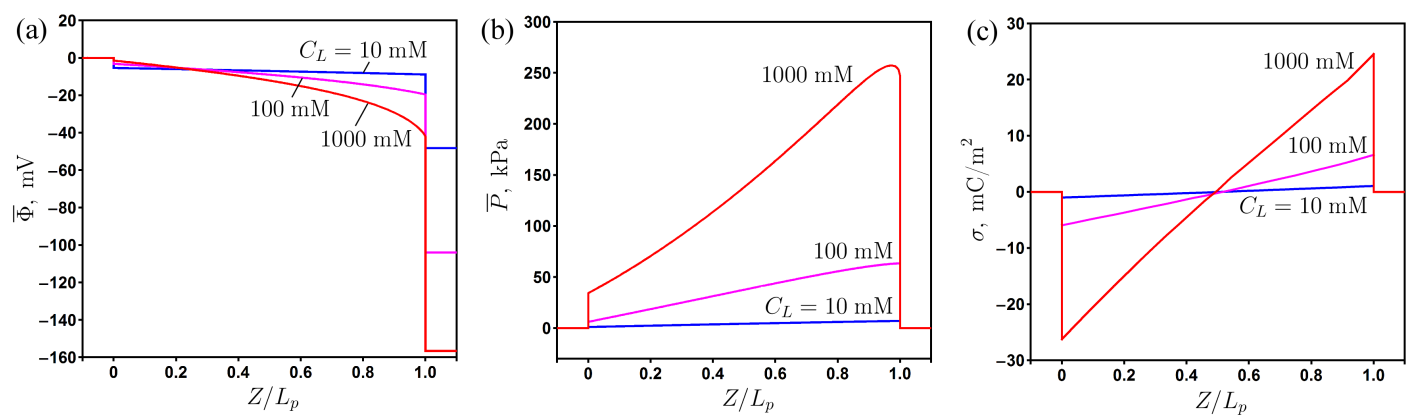

Figure 3: The cross-sectionally averaged potential (a), pressure (b), and surface charge density (c) for different concentrations $C_{L}$ at fixed $C_{R}=1 \mathrm{mM}$ in aqueous $\mathrm{LiOH}$ electrolyte.
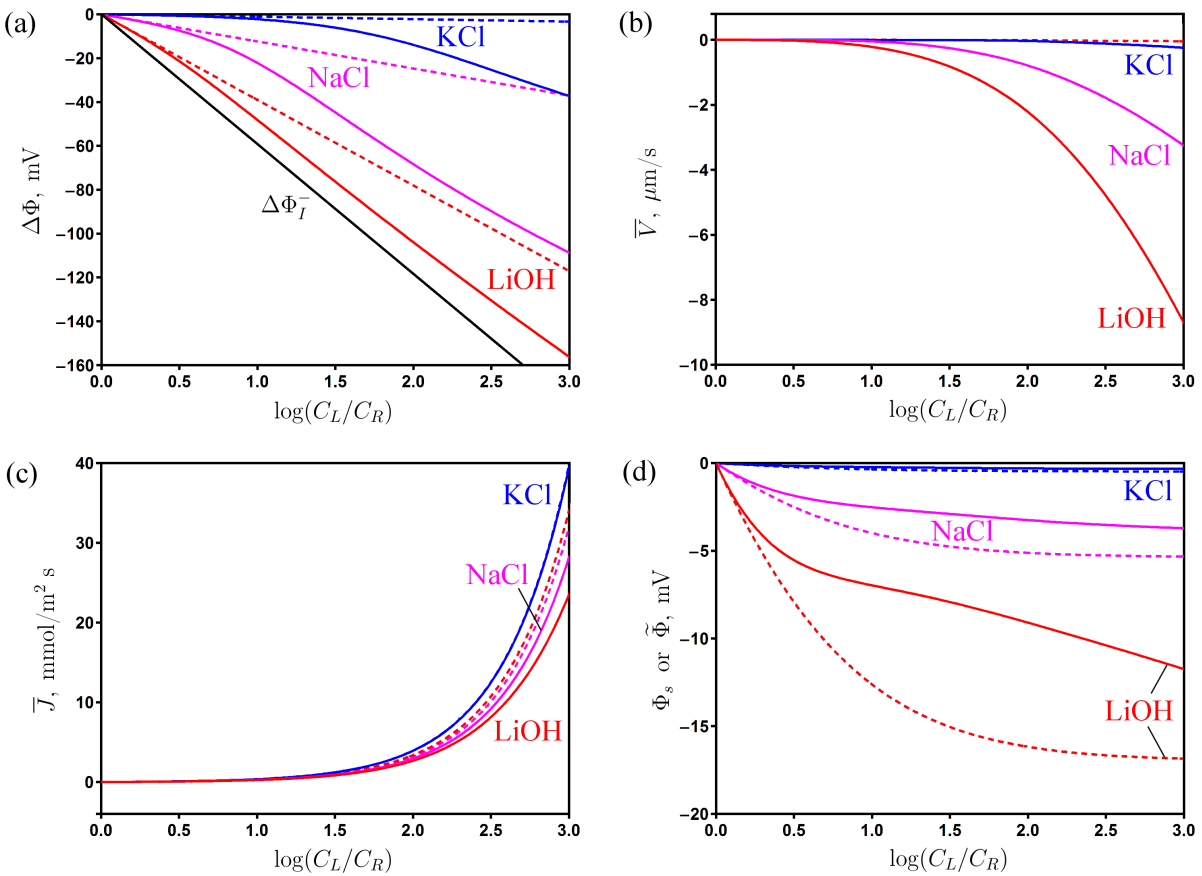

Figure 4: The membrane potential (a), average axial velocity (b), average ion flux (c), and surface $\Phi_{s}$ or pore-averaged $\widetilde{\Phi}$ potential (d) for different electrolytes. Non-polarizable nanopore (dashed curves) and polarizable nanopore (solid curves). $C_{R}=1 \mathrm{mM}$.

the relative enhancement of membrane potential in a polarizable nanopore in comparison with a non-polarizable one is close to that of $\mathrm{NaCl}$ electrolyte (around 2 times), compare Fig. 2 (a) and Fig. 2 (e).

For aqueous $\mathrm{LiOH}$ electrolyte, the contrast between ion diffusion coefficients is quite large $\left(D_{+} / D_{-}=0.195\right)$. It leads to the increase of induced charge (Fig. $\left.2(1)\right)$ and strong charge separation inside the nanopore, see Fig. 2 (j). The increase of concentration jumps at the pore entrance and exit results in the increase of osmotic pressure jumps, Fig. $2(\mathrm{k})$. The magnitude of membrane potential increases as well, but its relative enhancement in a polarizable nanopore with respect to a non-polarizable one becomes smaller in comparison with $\mathrm{KCl}$ and $\mathrm{NaCl}$ electrolytes, 
see Fig. 2 (i).

The effect of electrolyte concentration $C_{L}$ in the left reservoir on the averaged potential, averaged pressure, and induced charge profiles is demonstrated in Fig. 3. When the concentration $C_{L}$ increases at fixed $C_{R}$, the diffusive fluxes of cations and anions become larger (second term in the right-hand side of Eq. (1)), so a larger electric field (third term in the right-hand side of Eq. (1)) is required to make the total fluxes equal. It results in a stronger induced surface charge, higher osmotic pressure jumps, and larger magnitude of membrane potential.

The dependence of membrane potential for different electrolytes on the logarithm of concentration ratio $C_{L} / C_{R}$ at fixed $C_{R}=1 \mathrm{mM}$ is presented in Fig. 4 (a). When this ratio increases, the enhancement of membrane potential in a polarizable nanopore in comparison with a non-polarizable one becomes stronger, see also Fig. 3 (a). The increase of averaged osmotic velocity with increasing $C_{L} / C_{R}$ and also in the sequence $\mathrm{KCl} \rightarrow \mathrm{NaCl} \rightarrow \mathrm{LiOH}$ is demonstrated in Fig. 4 (b). It can be explained by the increased pressure gradient along the pore in both cases, see Fig. 3 (b) and Fig. 2 (c,g,k), respectively. In a non-polarizable pore, there is no osmotic flow due to the absence of osmotic pressure gradients. A larger concentration difference between the reservoirs leads to higher values of averaged ion fluxes, see Fig. 4 (c) and also Eq. (1). For a non-polarizable pore, the fluxes slightly decrease in the sequence $\mathrm{KCl} \rightarrow \mathrm{LiOH} \rightarrow$ $\mathrm{NaCl}$, which corresponds to the decrease of average electrolyte diffusion coefficient calculated as $2 /\left(1 / D_{+}+1 / D_{-}\right)[22]$. For a polarizable pore, the $\mathrm{LiOH}$ electrolyte demonstrates the lowest flux instead of $\mathrm{NaCl}$ electrolyte, probably, due to the presence of stronger osmotic flow in the direction from lower to higher concentration, see Fig. 4 (b). The polarizable pore also demonstrates the increase of surface potential with increasing the concentration contrast as well as in the sequence $\mathrm{KCl} \rightarrow \mathrm{NaCl} \rightarrow \mathrm{LiOH}$, see Fig. 4 (d). It can be explained by the stronger electric field, which develops inside the nanopore in these cases, see Fig. 3 (a) and Fig. 2 (a,e,i), respectively. In a non-polarizable nanopore, the absence of electric field suppression by the induced charge results in the higher values of pore-averaged potential, Fig. 4 (d).

\section{Conclusion}

In this work, we have studied theoretically the diffusion of binary aqueous electrolytes through nanopores with dielectric as well as conductive surface. The ion transport is described by the Space-Charge model, which is extended to the case of a polarizable nanopore wall with constant potential. It is shown that the diffusion of ions with different mobilities generates the electric field, which induces non-uniform surface charge in a polarizable nanopore. It results in charge separation inside the pore and leads to a dramatic enhancement of membrane potential in comparison with a non-polarizable nanopore. The calculations reveal that the induced charge and charge separation inside the polarizable nanopore become larger in the sequence $\mathrm{KCl} \rightarrow \mathrm{NaCl}$ $\rightarrow \mathrm{LiOH}$, which corresponds to the decrease of ion diffusion coefficients ratio from unity towards zero. The same trend is found for membrane potential, averaged osmotic velocity, ion fluxes, and pore surface potential. These quantities increase significantly with increasing the concentration contrast between the pore entrance and exit. The described phenomena may find applications in precise determination of ion mobilities, electrochemical and bio-sensing, as well as design of nanofluidic and bioelectronic devices.

This work is supported the Russian Science Foundation, Project 15-19-10017. 


\section{References}

[1] H. Strathmann, Introduction to membrane science, Wiley-VCH, 2011.

[2] Y. Tanaka, Ion Exchange Membranes: Fundamentals and Applications, Elsevier, Amsterdam, 2015.

[3] A.B. Yaroslavtsev, V.V. Nikonenko, V.I. Zabolotsky, Ion transfer in ion-exchange and membrane materials, Russ. Chem. Rev. 72 (5) 393-421 (2003).

[4] T. Teorell, An attempt to formulate a quantitative theory of membrane permeability, Proc. Soc. Exp. Biol. Med. 33 (1935) 282-285.

[5] K.H. Meyer, J.F. Sievers. La perméabilité des membranes I. Théorie de la perméabilité ionique. Helv. Chim. Acta 19 (1936) 649-664.

[6] A.H. Galama, J.W. Post, H.V.M. Hamelers, V.V. Nikonenko, P.M. Biesheuvel, J. Membr. Sci. Res. 2 (2016) 128-140.

[7] R.J. Gross, J.F. Osterle, Membrane transport characteristics of ultrafine capillaries. J. Chem. Phys. 49 (1968) 228-234.

[8] P.B. Peters, R. van Roij, M.Z. Bazant, P.M. Biesheuvel, Analysis of electrolyte transport through charged nanopores. Phys. Rev. E 93 (2016) 053108.

[9] G.B. Westermann-Clark, J.L. Anderson, Experimental verification of the space-charge model for electrokinetics in charged microporous membranes. J. Electrochem. Soc. 130 (1983) 839-847.

[10] G.B. Westermann-Clark, C.C. Christoforou, The exclusion-diffusion potential in charged porous membranes, J. Electroanal. Chem. 198 (1986) 213-231.

[11] M. Nishizawa, V.P. Menon, C.R. Martin, Metal nanotubule membranes with electrochemically switchable ion-transport selectivity, Science, 268 (1995) 700-702.

[12] C.R. Martin, M. Nishizawa, K. Jirage, M. Kang, S.B. Lee, Controlling ion-transport selectivity in gold nanotubule membranes, Adv. Mater. 13 (2001), 1351-1362.

[13] I.I. Ryzhkov, A.V. Minakov, Theoretical study of electrolyte transport in nanofiltration membranes with constant surface potential / charge density, J. Membr. Sci. 520 (2016) $515-528$.

[14] I.I. Ryzhkov, A.V. Minakov, Finite ion size effects on electrolyte transport in nanofiltration membranes. J. Sib. Fed. Univer.: Math. \& Phys., 10 (2017) 186-198.

[15] M.Z. Bazant, M.S. Kilic, B.D. Storey, A. Ajdari. Towards an understanding of inducedcharge electrokinetics at large applied voltages in concentrated solutions, Adv. Colloid Interface Sci. 152 (2009) 48-88.

[16] M.Z. Bazant, T.M. Squires, Induced-charge electrokinetic phenomena, Curr. Op. Coll. Inter. Sci. 15 (2010) 203-213. 
[17] C. Zhao, Y. Song, C. Yang. Induced-charge electrokinetics in a conducting nanochannel with broken geometric symmetry: towards a flexible control of ionic transport, Phys. Fluids 27 (2015) 012003.

[18] I.I. Ryzhkov, D.V. Lebedev, V.S. Solodovnichenko, A.V. Shiverskiy, M.M. Simunin, Induced-charge enhancement of diffusion potential in membranes with polarizable nanopores, Phys. Rev. Lett. 119, 226001 (2017).

[19] Ryzhkov I.I., Lebedev D.V., Solodovnichenko V.S., Minakov A.V., Simunin M.M. On the origin of membrane potential in membranes with polarizable nanopores. J. Membrane Science, 2018. V. 549. P. 616-630.

[20] V.S. Solodovnichenko, D.V. Lebedev, V.V. Bykanova, A.V. Shiverskiy, M.M. Simunin, V.A. Parfenov, I.I. Ryzhkov, Carbon coated alumina nanofiber membrane for selective ion transport, Adv. Engineer. Mater. 20 (2017) 1700244.

[21] S. Levine, J.R. Marriott, G. Neale, N. Epstein, Theory of electrokinetic flow in fine cylindrical capillaries at high zeta-potentials, J. Coll. Inter. Sci. 52, 136 (1975).

[22] E.L. Cussler, Diffusion in liquids. Cambdridge University Press, 2009.

\section{Теоретическое исследование диффузии электролитов че- рез поляризуемые нанопоры}

\section{Илья И. Рыжков, Антон С. Вяткин, Андрей В. Минаков}

Проведено теоретическое исследование дифбузии бинарных электролитов через нанопоры с диэлектрической, а также проводлщей поверхностъю на основе модели пространственного зарлда. Данная модель обобщена на случай поляризумой стенки поры с постоянным потенциалом. Показано, что диффузия ионов с различными подвиэностлми приводит к возникновению электрического поля, которое индуцирует неравномерное распределение заряда на поверхности проводящей поры. Это вызывает разделение заряда внутри поры и приводит к значительному увеличению мембранного потеницала по сравнению со случаем диэлектрической поры. Проведены расчеты для трех типов водных электролитов на основе $\mathrm{KCl}, \mathrm{NaCl}$ и LiOH. Исследовано влияние типа электролита и трансмембранной разности концентраций на перенос ионов и мембранньй потенциал.

Ключевые слова: нанопористая мембрана, перенос электролита, диффузия, индуцированный зарлд, двойной электрический слой, мембраннъй потенциал, численное моделирование 